\title{
Fault Diagnosis of Rolling Bearing using Deep Belief Networks
}

\author{
Tao Jie ${ }^{1,2, a}$, Liu Yi-Lun ${ }^{1,3, a}$,Yang Da-Lian ${ }^{1}$, Tang Fang ${ }^{1}$, and Liu Chi ${ }^{1}$ \\ ${ }^{1}$ College of Mechanical and Electrical Engineering, Central South University, Changsha 410083, China \\ ${ }^{2}$ Key Laboratory of Knowledge Processing and Networked Manufacturing, Hunan University of Science and Technology, Xiangtan 411201, \\ China \\ ${ }^{3}$ Lighe Alloy Research Institute, Central South University, Changsha 410083, China \\ ${ }^{a}$ Corresponding author
}

\begin{abstract}
This paper presents an approach to implement vibration signals for fault diagnosis of the rolling bearing. Due to the noise and transient impacts, it is difficulty to accurately diagnosis the faults with traditional methods. So a new type of learning architecture for deep generative model called deep belief networks (DBN) is applied. Since the unsupervised learning ability in DBN, it can extract the features from the raw data layer by layer. This article mainly research how to construct the encoder using DBN which can minimize the energy between the output and input vibration signals. Compared with existing diagnosis techniques, the proposed method can learn a good representation of features with higher accuracy. The results show that DBN can more comprehensively retain the data features in pattern recognition.
\end{abstract}

\section{Introduction}

The rolling bearing is widely used in actual engineering. According to the statistics, about $30 \%$ rotating machinery faults are related to rolling bearing damage. In bearing fault diagnosis, the most principal and useful method is vibration signal analysis. Due to the diversity and nonlinearity of vibration signals, the signal processing is the primal problem in rolling bearing fault diagnosis, it directly affects the fault diagnosis accuracy[1]. In recent years, people mainly adopt the methods in the time domain, frequency domain, time-frequency to extract the fault characteristics of vibration signals [2,3]. These methods have obtained the certain effect in fault diagnosis of rolling bearing. But it is difficult to eliminate the influence of false signals and data pollution.

Recently, the deep belief networks (DBN) has developed into a prevalent approach in machine learning for its prominent advantages such as the ability of unsupervised learning, fast inference and multi-layer structures. As a new kind of machine learning model, DBN primarily simulate the human brain and extract the input data features layer by layer[4,5,6]. Now the DBN has been successfully applied to mechanical fault diagnosis, text, voice, image recognition and other fields [7-10]. However, it has not been reported that using the DBN to reconstruct the vibration signals.

This paper develops a novel bearing fault diagnosis method using deep belief networks. The proposed diagnosis methodology can be structured in three stages: first, using the training samples to optimize the structure of DBN; second, developing DBN based on optimized structure for vibration signals reconstruction; third, implement the classifier to identify the testing samples. Rolling bearing fault diagnosis using DBN is compared with three existing diagnosis techniques: fast Fourier transform (FFT), Hilbert transform (HHT), Wavelet transform (WT). The results show that the proposed method can learn a good representation of features with higher accuracy.

\section{Bearing fault diagnosis using DBN}

This section details the proposed bearing fault diagnosis approach using DBN. The fault diagnosis process mainly included two parts: the signal reconstruction and faults recognition as shown in figure 1 .

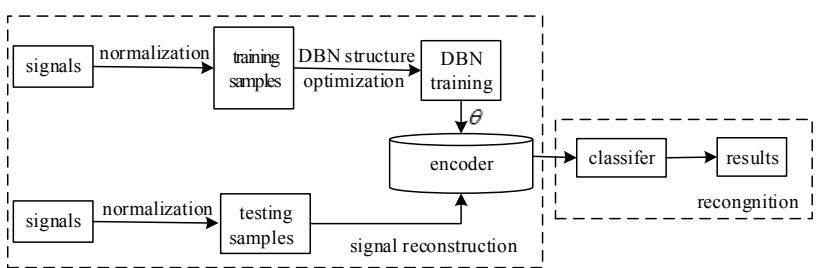

Figure 1. Fault diagnosis process based on DBN

In signal reconstruction, the vibration signals were collected from one rotate circle. And the signals were normalized into the data vectors to ensure DBN convergence. Then the training sets were used to practice the DBN and generated the encoder. For the test signals, it was put into the encoder and get the reconstituted vectors. In the recognitions, the feature vectors were put into the classifiers and produce the fault results.

\subsection{Deep belief networks}

The DBN is a kind of probability generation model, which produce the largest probability eligible sample. There is a BPNN in the top of DBN, and multiple hidden layers in the middle. The DBN employs a hierarchical structure with multiple stacked restricted Boltzmann machines and works through a layer by layer successive learning process. Each RBM consists of two layers as visible $\left(v_{i}\right)$ and hidden $\left(h_{i}\right)$ layer. The connections between the nodes $v_{i}$ and $h_{i}$ are restricted. The process of transformation of data from visible layer to the hidden layer is finished through a sigmoid activation function based on the RBM learning rule. 


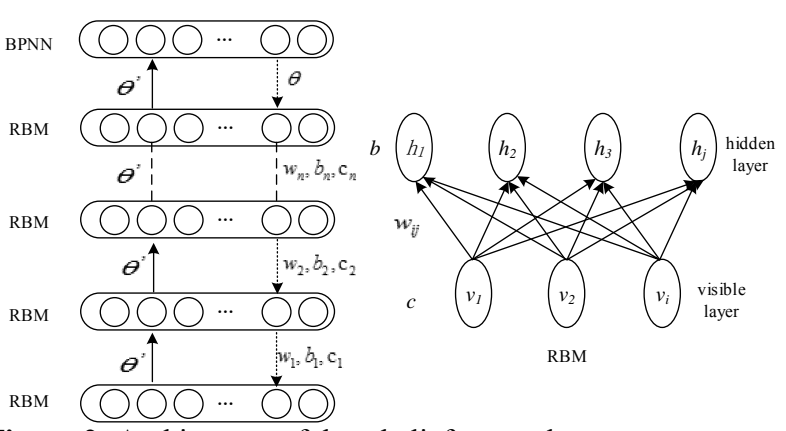

Figure 2. Architecture of deep belief networks

As shown in figure 2, the $v_{i}$ were the input layer nodes, $h_{i}$ were the output layer nodes, $w_{i j}$ were the weight from $v_{i}$ to $h_{j}, c$ were the input layer bias, $b$ were the output layer bias. The vector $b_{i}, c_{i}, w_{i j}$ constructed the parameter set $\theta$. The energy function and calculation formula of $v, h$ and $\theta$ were:

$$
\begin{aligned}
& E(\mathrm{v}, h)=h^{\mathrm{T}} w v+b^{\mathrm{T}} h+c^{\mathrm{T}} v \\
& P\left(h_{i}=1\right)=\frac{1}{1+\exp \left(-b_{j}-\sum_{i} v_{i} w_{i j}\right)} \\
& P\left(v_{i}=1\right)=\frac{1}{1+\exp \left(-c_{i}-\sum_{j} v_{j} w_{j i}\right)} \\
& \frac{\partial \log p\left(\mathrm{v}^{0}\right)}{\partial \theta_{i j}}=<v_{i}^{0} h_{j}^{0}>-<v_{i}^{\infty} h_{j}^{\infty}> \\
& \theta_{t+1}=\theta_{t}+\left.\eta \frac{\partial \log P(\mathrm{v}, \mathrm{h})}{\partial \theta}\right|_{\theta_{t}}
\end{aligned}
$$

$\mathrm{DBN}$ is constructed by stacking RBMs layer by layer. This architecture ensures that DBN can be practiced through the CD algorithm[4]. The DBN calculation process includes two major parts: the first procedure is called pre-training, each RBM layer is trained by using the activation probabilities of the lower-layer RBM as the input training data; the second procedure is fine-tune, the back-propagation algorithm can be used to adjust the network weights.

\subsection{DBN structure Optimization}

DBN structural optimization mainly determine the layer number, nodes number, learning rate, batch size etc. Because the sizes of fault signal is different, they need different structure model to adapt the feature extraction. The figure 3 is the flow chart of structure parameter optimization in DBN.

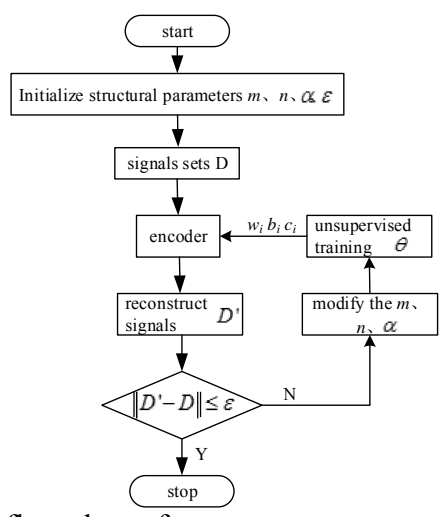

Figure 3. The flow chart of structure parameter optimization

In the structure parameter adjustment, given an vibration signals, the encoder minimizes the energy between the output and input vectors using function (1). In the unsupervised learing, DBN trained the first hidden layer, and saved the training result as higher (more abstract) layer of input. Then the DBN was trained from bottom to up, and formated the model parameter vector group $\theta^{\prime}$. Then using the BP algorithm fine-tune the model parameters $\theta^{\prime}$ from top to bottom. The paraments $\theta$ ' assigned to $w_{i}, b_{i}, c_{i}$ one by one. Using the $w_{i}, b_{i}, c_{i}$ calculat the singnals' input and output vector. When the signal reconstruction error was less than the threshold, the cycle was terminated, the algorithm was as follows:

(1)Initialized the DBN structure parameters: hidden node number $m$, hidden layer number $n$, reconstruct error epsilon rate $\varepsilon$.

(2)Input the signal sets D, and the signal D' was generated by the encoder.

(3)The reconstrct error were algorithmed by $\left\|D^{\prime}-D\right\|$.

(4)If the reconstrct error is less or equal to the $\varepsilon$, save the $\theta$ and generate the encoder, othersize modify the parameters $m, n$ and unsurpsived training the DBN to update the $\theta$.

\section{Experimental verification}

\subsection{Test cases}

To test and verify the feasibility of proposed method, this article adopted the bearing fault experimental data from case western reserve university. The bearings was produced by SKF bearing 6205-2 deep groove ball bearings, sampling frequency was $12000 \mathrm{~Hz}$, motor speed was $1797 \mathrm{rpm}$. The experiment selected the normal bearing, inner-ring faults and rolling body fault to identify the rolling bearing faults. Single point faults were introduced to the test bearings using electrodischarge machining with fault diameters of $0.18 \mathrm{~mm}$. The sampling time was 4 second and the data points was 48000. According to the sampling frequency and motor speed, the specimen rotated 400 sampling points as a data vector. The original signal waveform of various states bearing as shown in figure 4 . 


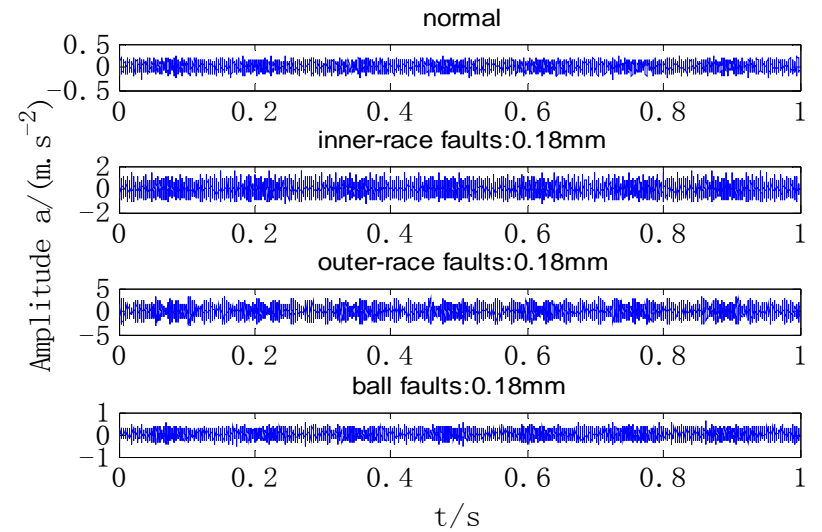

Figure 4. The original signal waveform of bearing

As shown in figure 4, the signals were collected from three types fault signals and one normal signals. And it was difficult to identify the rolling bearing faults from such vibration signals.

\subsection{Signal reconstruction using DBN}

In this experiment, the 100 groups sample were selected from each fault sample set, then to construct the training sample matrix of $400 \times 400$. For the parameters initialization, the threshold of reconstruct error was 0.1 , The parameters $m, n$ initialized by 50 and 1 . The model calculated the signals reconstruct error, if the error was less than 0.1 , generate the encoder. If the error was more than 0.1 , increased the $m$ and $n$, and update the $\theta$ by the unsurprised training in DBN, until the error was less than 0.1. Through the DBN structure optimization, the structure of DBN was shown in Table 1.

Table 1. The structure of DBN

\begin{tabular}{cc}
\hline Parameters & Value \\
\hline Number of input nodes & 428 \\
Number of iterations & 1 \\
The activation function & Sigmoid \\
Mini-batch size & 100 \\
Number of hidden layers & 3 \\
Number of neurons in a layer & 200 \\
The reconstruction error & 0.1 \\
Learning rate & 0.78 \\
\hline
\end{tabular}

According to the structure parameter adjustment, the hidden layer increased from 1 to 3 , the hidden nodes increased from 50 to 200 . So there were three RBMs in the DBN, every RBM had a parameter set including $w_{i}$, $b_{i}, c_{i}$, as is shown in table 2 .
Table 2. Some parameters of encoder

\begin{tabular}{cccccc}
\hline Parameters & \multicolumn{5}{c}{ Value } \\
\hline$w_{1}$ & -0.1945 & -0.2229 & -0.2083 & -0.2195 & $\ldots \ldots$ \\
& -0.2032 & -0.2338 & -0.2315 & -0.2180 & $\ldots \ldots$ \\
$b_{1}$ & -1.2859 & -1.3292 & -1.4016 & -1.3114 & $\ldots \ldots$ \\
$c_{1}$ & -0.0888 & -0.0681 & -0.1472 & -0.2208 & $\ldots \ldots$ \\
& -0.1758 & -0.1891 & -0.2144 & -0.2271 & $\ldots \ldots$ \\
$w_{2}$ & -0.1855 & -0.1986 & -0.2236 & -0.2356 & $\ldots \ldots$ \\
& -0.0928 & -0.2384 & -0.5229 & -0.3611 & $\ldots \ldots$ \\
$b_{2}$ & -0.5318 & -0.6009 & -0.5432 & -0.6075 & $\ldots \ldots$ \\
$c_{2}$ & -0.0665 & -0.0549 & -0.0573 & -0.0606 & $\ldots \ldots$ \\
& -0.0637 & -0.0527 & -0.0566 & -0.0580 & $\ldots \ldots$ \\
$w_{3}$ & -0.2259 & -0.2560 & -0.2874 & -0.2457 & $\ldots \ldots$ \\
$b_{3}$ & -0.0126 & 0.0773 & 0.0229 & -0.0217 & $\ldots \ldots$ \\
$c_{3}$ & & & & & \\
\hline
\end{tabular}

As shown in table 2, the experiment generated the three hidden layers DBN encoder which used to extract the test signal characteristic information. Among them, the $w_{1}, b_{1}$ and $c_{1}$ were the coding parameters in the first hidden layer $\left(\mathrm{RBM}_{1}\right)$, the $w_{2}, b_{2}$ and $c_{2}$ were the coding parameters in the second hidden layer $\left(\mathrm{RBM}_{2}\right)$, the $w_{3}, b_{3}$ and $c_{3}$ were the coding parameters in the third hidden layer $\left(\mathrm{RBM}_{3}\right)$. Using the formula (1) and (2) calculated the signal feature value layer by layer. Then we got the reconstitution signal as shown in figure 5 .

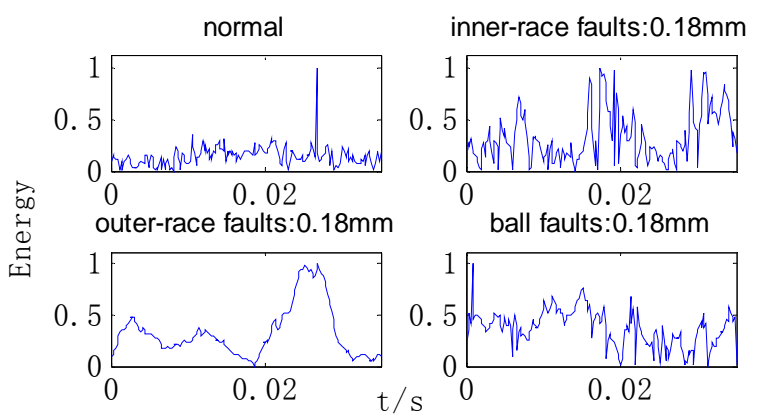

Figure 5. The time waveform of test signals under DBN feature abstract

The test signal was input to the encoder and generate the reconstitution signal. As shown in figure 5, the four types vibration signals were reconstructed by the encoder. Compared the figure 4 and figure 5 , the reconstructed signal dimension has fell from 428 to 200 . The feature waveform not only retained the fault signal energy shock, cycle and amplitude, but also recorded the energy change point. So we could use the reconstitution signal to describe the fault type and damage.

\subsection{Results and analysis}

In the reconginiton, we used the $\mathrm{KNN}$ classifier to identify all kinds of fault signal. As sufficient as possible for the singnals' information, the expriment selected 14 indexes as fault feature such as the signal sample average maximum, minimum, kurtosis, tie, the peak - peak value, amplitude value, variance, standard deviation, RMS, waveform factor and peak factor, pulse factor, margin factor. Compared with Wavelet-3, HTT and FFT means, the results were shown in table 3 . 
Table 3. Results of bearing fault diagnosis

\begin{tabular}{ccc}
\hline Methods & Computation time $/ \mathrm{s}$ & Accuracy $/ \%$ \\
\hline DBN & 19.26 & 96.67 \\
Wavelet-3 & 20.13 & 91.67 \\
HHT & 18.57 & 93.33 \\
FFT & 15.79 & 86.67 \\
\hline
\end{tabular}

In the reconginitong, the accuracy of DBN was $96.67 \%$, it was higher than other methods. The computation time of DBN was longer than HHT and FFT, but shorter than Wavelet-3. In the DBN signal reconstruction, the singal calculated mainly through the hidden layers step by step, so the running time has increased with the hidden layers and nodes in the DBN. Compared with Wavelet-3, HTT and FFT means, the suggested methods were more effective and accurate for the identification of rolling bearing fault diagnosis in various situations.

Due to the good ability of unsupervised learning, the DBN can effectively extract the data characteristics and effectively avoid fitting or local extremum problems. In the parameter passed layer by layer, the useful information was effective to keep the layers and constantly improved in the next layer of feature extraction.

\section{The Conclusion}

This paper has presented the novel approach of rolling bearing fault diagnosis using the DBN to reconstruct the vibration signals. The proposed method is applied to analyze the experimental vibration signals of rolling bearing. Due to the unsupervised learning, DBN extracted the vibration signal characteristics layer by layer, so it could more comprehensively retain useful information of the original data. The results confirm that it performs better than other exiting methods. The future study will pay more attention to improving the calculation efficiency of the proposed method.

\section{References}

[1] W. Guobiao, H. Zhengjia, C. Xuefeng, et al. Basic research on machinery fault diagnosis-What is the prescription J. Journal of Mechanical Engineering, 49 (1) 63-72, (2013).

[2] Z. Jinde, C. Junsheng. Improved Hilbert-Huang Transform and Its Applications to Rolling Bearing Fault Diagnosis J. Journal of Mechanical Engineering, 51(1) 138-145, (2015).

[3] Z. Long, H. Wen-yi, X. Guo-liang. Assessment of rolling element bearing fault severity using multiscale entropy J. Jouranal of Vibration and Shokc, 33(9), 185-189 (2014).

[4] G. E Hinton, S. Osindero, Y. W Teh. A fast learning algorithm for deep belief nets J.Neural Computation, 18(7), 1527-1554, (2006).

[5] Y. Bengio, P. Lamblin, D. Popovici, et al. Greedy layer-wise training of deep networks J. Advances in neural information processing systems, 19, 153-160, (2007).
[6] J. Schmidhuber. Deep learning in neural networks: An overview J. Neural Networks, 61(1), 85-117, (2015).

[7] T. Tran V, F. Althobiani, Ball A. Anapproach to fault diagnosis of reciprocating compressor valves using Teager-Kaeser energy operator and deep belief networks J. Expert Systems with Application, 41(9), 4113- 4122, (2014).

[8] J. Sun, R. Wyss, A. Steinecker, et al. Automated fault detection using deep belief networks for the quality inspection of electromotors $\mathrm{J}$. Technisches Messen, 81(5), 255- 263, (2014).

[9] P. Tamilselvan, W. Yibin, W. Pingfeng. Deep belief network based state classification for structural health diagnosis J. Reliability Engineering and System Safety, 115(3), 124- 135, (2013).

[10]L. Yan-feng, W. Xin-qing, Z. Mei-jun, et al. An Approach to Fault Diagnosis of Rolling Bearing Using SVD and Multiple DBN Classifiers J. Journal of Shanghai Jiao Tong University, 49(5), 681- 686, (2015). 